\title{
First Case of Successful Implantation and Live Birth after Double Trophectoderm Biopsy before and after Vitrification on the Same Cohort of Blastocyst
}

\author{
${ }^{1}$ Harsha Bhadarka, ${ }^{2}$ Nayana H Patel, ${ }^{3}$ Yuvraj D Jadeja, ${ }^{4}$ Kruti B Patel, ${ }^{5}$ Niket Hitesh Patel, ${ }^{6}$ Molina N Patel
}

\begin{abstract}
Introduction: To report the first case of live birth after double trophectoderm biopsy before and after vitrification on the same cohort of blastocyst in our knowledge.
\end{abstract}

Design: Case report.

Patient: A 36-year-old female with a history of 13 years of active married life for treatment of infertility.

Main outcome measure: Live birth after double trophectoderm biopsy.

Results: Double biopsy pre- and postvitrification and its positive outcome.

Conclusion: Preimplantation genetic screening and diagnosis (PGS/PGD), though an invasive procedure on the embryos, when done meticulously would not dampen the implantation potential of the embryo and second biopsy could be a feasible option to salvage embryos with inconclusive or suspected falsepositive PGS/PGD reports.

Keywords: Blastocyst, Double trophectoderm biopsy, In vitro fertilization/intracytoplasmic sperm injection, Preimplantation genetic screening and diagnosis, Vitrification.

How to cite this article: Bhadarka $\mathrm{H}$, Patel $\mathrm{NH}$, Jadeja $\mathrm{YD}$, Patel KB, Patel NH, Patel MN. First Case of Successful Implantation and Live Birth after Double Trophectoderm Biopsy before and after Vitrification on the Same Cohort of Blastocyst. Int J Infertil Fetal Med 2017;8(3):120-124.

Source of support: Nil

Conflict of interest: None

\footnotetext{
${ }^{1}$ Lab Director, ${ }^{2,5}$ Gynecologist and Medical Director, ${ }^{3,6}$ Gynecologist ${ }^{4}$ Senior Embryologist

${ }^{1}$ Department of IVF, Akanksha Hospital \& Research Institute Anand, Gujarat, India; Department of Biochemistry, P.D. Patel Institute of Applied Sciences, Anand, Gujarat, India

${ }^{2,3,5,6}$ Department of Obstetrics and Gynecology, Akanksha Hospital \& Research Institute, Anand, Gujarat, India

${ }^{4}$ Department of IVF, Akanksha Hospital \& Research Institute Anand, Gujarat, India

Corresponding Author: Harsha Bhadarka, Lab Director Department of IVF Akanksha Hospital \& Research Institute Anand, Gujarat, India; Department of Biochemistry, P.D. Patel Institute of Applied Sciences, Anand, Gujarat, India, Phone: +919727416492, e-mail: harshabhadarka@yahoo.co.in
}

\author{
Date of received: 19/07/2017
}

Date of acceptance: 07/08/2017

Date of publication: November 2017

\section{INTRODUCTION}

Preimplantation genetic diagnosis and screening for monogenic diseases and/or numerical/structural chromosomal abnormalities is a tool for embryo testing aimed at identifying euploid embryo in cohort produced during in vitro fertilization (IVF) cycle. ${ }^{1}$ The aim of PGS/PGD is to define whether an embryo is affected by monogenic disease and/or chromosomal impairment, thus preventing implantation of an abnormal fetus limiting the risks underlying the transfer of a chromosomally abnormal embryo. In recent years, PGD/PGS has gained a lot of momentum; various studies supporting and an equally good number opposing its role in IVF outcomes have been published. Preimplantation genetic screening and diagnosis in recent times has also come under a lot of criticism due to lot of ethical as well as scientific reason, but there is no doubt that PGD/PGS has been one of the most talked about important breakthrough in IVF. A critical aspect of this technology is the possibility that the biopsy itself can adversely affect the quality of embryo. Different approaches to biopsy have been proposed. Cleavage stage biopsy of the blastomere was the mostly commonly accepted approach. Polar body biopsy, cleavage stage biopsy, and trophectoderm biopsy all have been probed in detail for its advantages and disadvantages. Polar body biopsy either sequential or simultaneously was encouraged as an alternative to blastomere biopsy due to two significant reasons. First is the ethical and legal reason where in some countries where embryo biopsy is not allowed, it remains the obvious choice and only option, and second is due to the fact that polar body biopsy is comparatively less invasive than cleavage or trophectoderm biopsy. Cleavage stage biopsy is normally performed on day 3 on embryos with at least six blastomeres. Kirkegaard et al ${ }^{2}$ compared the blastocyst development of biopsied day 3 embryo with that of nonbiopsied embryo and showed that biopsied embryo showed delayed compaction process and hatched in nonphysiological fashion, resulting in small blastocyst 
with thicker zona pellucida. Single blastomere biopsy again raises question of diagnosing mosaicism and more than one blastomere would lead to reduction in embryonic mass of about $25 \%$ impacting clinical outcome. ${ }^{3,4}$ The fragile nature of cleavage stage embryo and the fact that the embryonic genome activation would have not occurred till then further raised question on the cleavage stage being optimal time of embryo biopsy. Autocorrection of embryo till blastocyst and lower blastocyst rate after cleavage stage biopsy as opposed to undisturbed embryos again raised serious doubt on cleavage stage biopsy. Blastocyst biopsy was an important breakthrough in modern-day IVF reported first by Boer et $\mathrm{al}^{5}$ and the first live birth was by Kokkali et al. ${ }^{6}$ Presently, due to various reasons, trophectoderm biopsy is replacing both cleavage stage and polar body biopsy. Trophectoderm biopsy due to its technical compliance and biological plausibility soon became a standard approach in embryology for PGD/PGS. Strict and higher standards in culture system as well as precise cryopreservation technique have made widespread implementation of this procedure an important limiting factor. Two different approaches have been published. ${ }^{3}$ First, a hole in zona on day 3 and second ${ }^{7}$ approach entails simultaneous zona opening and trophectoderm biopsy on an undisturbed embryo on days 5, 6, or even 7. Many European institutes still hold on to their cleavage stage approach for their own reasons. The exact effect on biopsy is yet to be evaluated and still remains unknown. Multiple times in recent years, the effect of biopsy by breaching the embryo has been raised. There is a group of biopsy experts who still firmly believe in this procedure to harm the developing embryo. Here, in our knowledge, we are reporting the first case of live birth after double trophectoderm biopsy before and after vitrification, bringing to light the fact that if meticulously performed, the implantation potential of the embryo would not be compromised.

\section{MATERIALS AND METHODS}

A 36-year-old female and a 39-year-old man approached our center (Akanksha Hospital and Research Institute) with a history of 13 years of active married life for treatment of infertility. Seminal fluid examination according to World Health Organization 2010 recommendation was normal. Her basic workup and endocrine profile were normal. Antral follicle count was 12 and anti-Müllerian hormone was $5 \mathrm{ng} / \mathrm{mL}$. She was a known case of polycystic ovary syndrome with history of polycystic ovary drilling in the past. She also had a history of genital tuberculosis diagnosed by endometrial tuberculosis polymerase chain reaction (PCR) confirmed on culture. She had completed anti-Koch's treatment for the same.
She was G4A2L0E2, all conceived through a sum total of six fresh IVF cycle and one frozen, out of which four times she conceived leading to two missed abortion and two ectopic as mentioned earlier and the rest were failed cycles. She had to undergo laparoscopy twice for the treatment of ectopic pregnancies. After detailed evaluation of her case, decision to go for an IVF cycle with her own eggs followed by blastocyst transfer after PGS along with time-lapse imaging technique embryoscope was made. Informed written consent was taken from the couple for publication of the case. She was stimulated from day 2 of her cycle with antagonist protocol. On her basal day 2, luteinizing hormone ( $\mathrm{LH})$ was $2.3 \mathrm{mIU} / \mathrm{mL}$ and estradiol (E2) was $66.73 \mathrm{pg} / \mathrm{mL}$. She weighed $70 \mathrm{~kg}$. A single dose of antagonist (injection Cetrotide $0.25 \mathrm{mg}$, Merck Serono) was given on day 1 of stimulation along with $300 \mathrm{IU}$ recombinant follicle-stimulating hormone $(\mathrm{rFSH}$; Injection gonal F, Serono). On day 5, folliculometry was performed and antagonist was added again as per the follicular growth. On day 7 of her cycle, $\mathrm{rFSH}$ was made 225 IU and 75 IU recombinant LH (injection Luveris, Merck Serono) was added along with antagonist. On day 10, her E2 was 6354.56 and her LH was 2.49 and progesterone was $1.78 \mathrm{ng} / \mathrm{mL}$ so to prevent the chances of hyperstimulation trigger with agonist injection leuprolide and acetate, $4 \mathrm{mg}$ (Inj Leuprogen, LG life sciences) was made and egg retrieval done 36 hours later. Egg retrieval was done under short general anesthesia and was uneventful. Thirteen retrieved oocytes were washed and incubated in continuous single culture (CSC; Irvine Scientific, California, USA) for around 2 hours at $37^{\circ} \mathrm{C}$ in atmosphere of $5 \% \mathrm{CO}_{2}$ in Heracell incubator. Semen sample was washed using a flushing medium (Medicult, Denmark) followed by swim up method. Prewashed count was 28 million $/ \mathrm{mL}$ and $30 \%$ motility, afterwash count was 4 million $/ \mathrm{mL}$ and $98 \%$ motility. The oocytes were denuded of their surrounding cumulus cells approximately 2 hours after retrieval using hyaluronidase $80 \mathrm{IU} / \mathrm{mL}$ buffered with gentamicin (Irvine Scientific, California, USA) for 10 to 15 seconds. The oocytes were then transferred to same CSC medium for complete mechanical removal of the cumulus cells with sterile pulled glass Pasteur pipettes. The oocytes were rinsed and incubated for 1 hour in CSC medium under light mineral oil (Irvine Scientific, California, USA). Out of 13 oocytes, 10 were mature and 1 was immature and 2 were germinal vesicle. Intracytoplasmic sperm injection (ICSI) was done on mature and immature oocytes and were incubated in embryoscope (Unisense FertiliTech, Denmark) containing $6 \% \mathrm{CO}_{2}, 5 \% \mathrm{O}_{2}$, and $89 \% \mathrm{~N}_{2}$ at $37^{\circ} \mathrm{C}$. Next day three oocytes with two pronuclei were seen. On day 3 at cleavage stage fresh medium was replaced in dish. All three embryos reached up to blastocyst stage. Trophectoderm biopsy dish was prepared with $10 \mu \mathrm{L}$ of Quinn's advantage medium with HEPES ( $\mathrm{Ca} / \mathrm{Mg}$ free) 

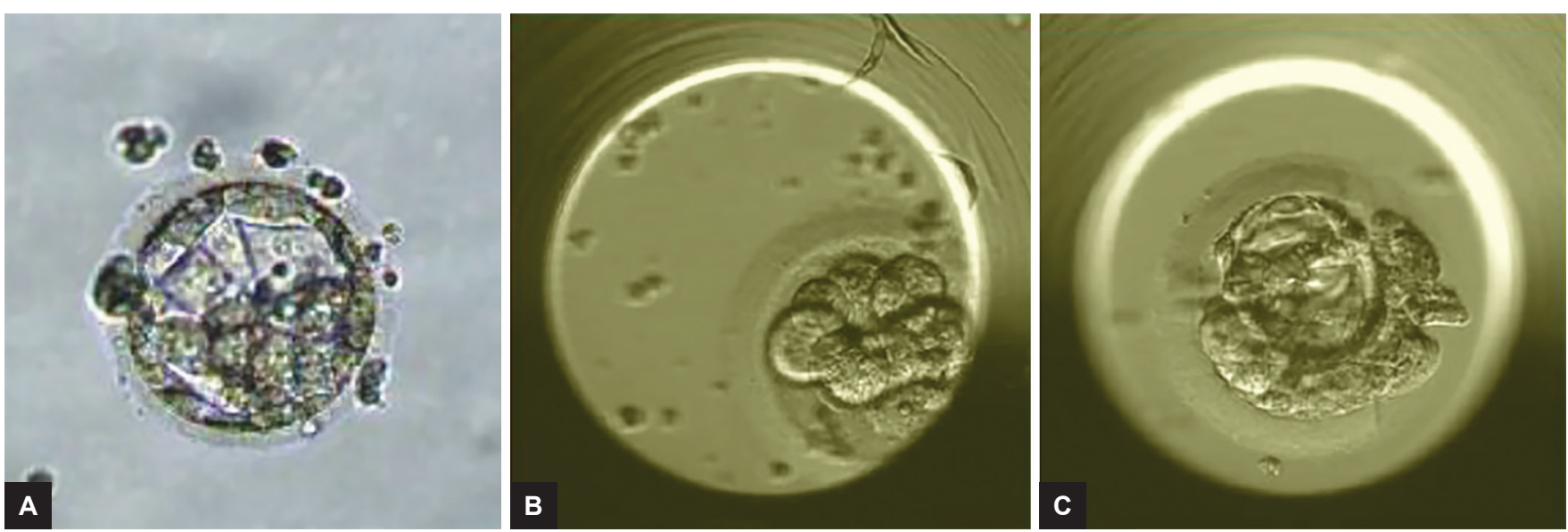

Figs 1A to C: After first-time biopsy: (A) NK1; (B) NK2; and (C) NK3 (Identification: NK)

(Sage, Connecticut, USA) under light mineral oil and subjected to biopsy. A hole was made in the zona pellucida (at 3 o'clock position) exactly opposite to the inner cell mass at 9 o'clock with the polar body at 6 o'clock of each blastocyst using LYKOS (instrument company name for lesser assisted hatching to zona pellucid) (Hamilton Thorne, Beverly, Massachusetts, USA). Applying gentle suction with the biopsy pipette (Origio, Denmark), trophectoderm cells were encouraged to herniate from the zona pellucida, five to seven trophectoderm cells were dissected from each of the blastocyst. The biopsied cells were placed in deoxyribonuclease-ribonuclease Free PCR tubes containing $2.5 \mu \mathrm{L}$ phosphate-buffered saline. All three biopsied embryos were then frozen because of clinical reasons of agonist trigger and the possibility of hyperstimulation and also due to rising levels of progesterone. Pre-implantation genetic screening was done using Next-genome sequencing method at Sat-Kaival Institute of Molecular Laboratory, Anand, India. On next day, PGS report showed two embryos to be noninformative and one embryo was abnormal (high confidence). Though PGS report was noninformative, both embryos were well developed and with precise morphokinetics (Fig. 1). Due to the noninformative reporting of the biopsy and optimal quality of embryo, decision to not discard the embryos was made and after thorough deliberation of the IVF team and embryology team and detailed counseling of the couple, decision to go for a repeat biopsy followed by frozen embryo transfer was made. The patient's endometrial preparation was started from day 3 of her cycle after transvaginal scan, confirming the endometrial thickness to be $3 \mathrm{~mm}$, and she was started on E2 valerate (T. Progynova $2 \mathrm{mg} /$ day, German Remedies Ltd.) till her endometrial thickness was $8 \mathrm{~mm}$ and then her estrogen supplementation was increased to $18 \mathrm{mg} /$ day with injection progesterone (injection Strone, Serum Institute of India Pvt Ltd) $50 \mathrm{mg} /$ day intramuscularly for 6 days. Repeat biopsy was performed precisely after thawing of embryo on day 6 of progesterone through same
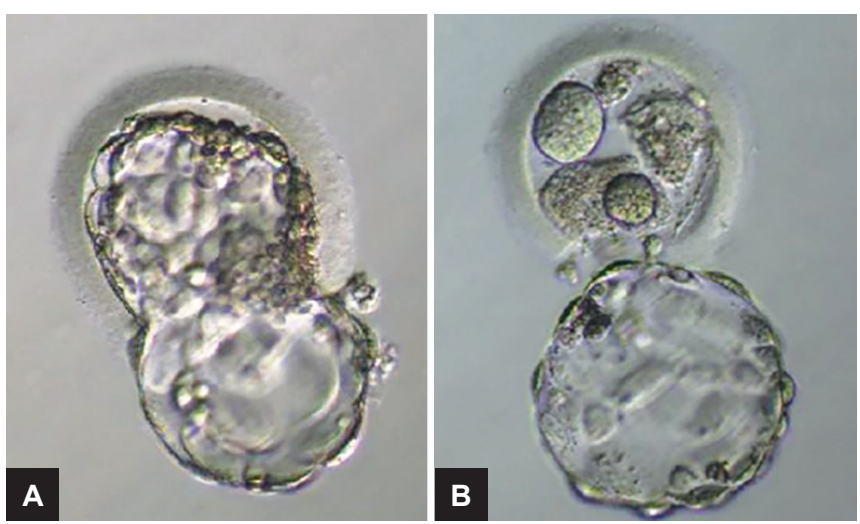

Figs 2A and B: Transferred embryo: (A) NK 1; and (B) NK2

hole, which was created by laser-assisted hatching during previous biopsy. The trophectoderm cells were again sent to genetic lab for PGS. On the next day, PGS report showed two normal embryos with high confidence. One hatched blastocyst (grade V: 1:1) and the other zona pellucida free mass (grade VI: 1:1) ${ }^{8}$ was carefully loaded in embryo transfer catheter (Cook Medical, USA) and transferred to patient's uterus under sonography guidance (Fig. 2).

\section{RESULTS}

After 15 days of embryo transfer, beta human chorionic gonadotropin (hCG) was 557.6, which indicated a positive outcome. The beta-hCG doubled after 48 hours as expected to 1,100.5. She was called for her first scan at 7 weeks, which showed single live intrauterine pregnancy, corresponding to weeks of gestation. She had complaints of bleeding per vaginum at 9 and 11 weeks of her pregnancy, which were conservatively managed. Her double marker and nuchal translucency scan at 12 weeks showed low risk for aneuploidy. Her anomaly scan at 22 weeks was normal; the rest of her pregnancy remained uneventful. She delivered a healthy male $2.6 \mathrm{~kg}$ at 37 weeks 4 days through elective lower segment cesarean section. 


\section{DISCUSSION}

Trophectoderm biopsy is an extremely delicate procedure, requiring high set of skills by the biologist who performs it. There always remains the question of embryos being damaged if not meticulously performed. An embryo requiring to be biopsied twice is an even rare entity and would require even higher care and caution on the part of the embryology laboratory and the person performing the biopsy. Case reports of the double biopsy on the same embryo one at cleavage stage followed by trophectoderm biopsy have been reported by Taylor et $\mathrm{al}^{9}$ and Wininger et al. ${ }^{10}$ A case of triple biopsy also has been reported where first polar body was biopsied followed by cleavage stage followed by trophectoderm biopsy, which resulted later in live birth. ${ }^{11}$ But this case to our knowledge is the first case of trophectoderm biopsy being performed twice on the same embryo before and after vitrification and leading to a successful implantation and birth of a healthy baby boy of $2.7 \mathrm{~kg}$. Effect of cleavage stage biopsy on the embryo in a recent randomized controlled trial demonstrated a lower implantation potential ${ }^{12}$ and decrease in embryonic mass by $25 \%$. But the effect of trophectoderm biopsy on the embryo and especially twice as in this case report remains unknown for obvious reason. The utilization of PGS for improving IVF outcomes on a more wider scale has come under a lot of criticism, ${ }^{13,14}$ along with various other studies which have strongly questioned the importance of PGS in improving IVF outcomes. But, i.e., an entire unanswered debate of recent times that we want to discuss here with two very important practical points. 1. The effect of this invasive procedure on the quality of embryos and its implantation potential.

2. The possibility of a second trophectoderm biopsy on a previously biopsied embryo for specific reasons, which could lead to rescuing precious embryos from being discarded.

This case report at least proves that if meticulously performed and under stringent and favorable embryology laboratory condition, trophectoderm biopsy would not really damage the implantation potential of the embryo as here inspite of the biopsy being performed twice, a positive implantation with live birth was achieved. Embryos with inconclusive PGS/PGD reports are usually not recommended for embryo transfer and could lead to discarding perfectly normal euploid embryos. Capalbo ${ }^{15}$ has strongly raised the issue of false high positive rate of aneuploid and mosaic embryos. In spite of such events being rare where PGS/PGD reports are inconclusive, every clinic providing PGS/PGD services would have faced such situation. The reason for this PGS report could be many, such as sampling or biopsy error, amplification artifacts, other procedural aspects, such as reagent batch and inadequate quality control of laboratory, and false data analysis. All these would lead to erroneous discarding of embryos with reproductive potential. A second biopsy could be the light of hope for these embryos to be salvaged and brought to life, saving the couple from physical and financial burden of repeated IVF cycles. Of course, more large studies would be needed to confirm the hypothesis put forward through this case. But this case definitely opens up a debate of not throwing away inconclusive PGS/PGD reported embryos or mosaic embryos and reconfirming its diagnosis through a second biopsy.

\section{REFERENCES}

1. Cimadomo D, Capalbo A, Ubaldi FM, Scarica C, Plalgiano A, Canipari R, Rienzi L. The impact of biopsy on human embryo developmental potential during preimplantation genetic diagnosis. BioMed Res Int 2016;2016:1-10.

2. Kirkegaard K, Hindkjaer JJ, Ingerslev HJ. Human embryonic development after blastomere removal: A time-lapse analysis. Hum Reprod 2012 Jan;27(1):97-105.

3. McArthur SJ, Leigh D, Marshall JT, De Boer KA, Jansen RPS. Pregnancies and live births after trophectoderm biopsy and preimplantation genetic testing of human blastocysts. Fertil Steril 2005 Dec;84(6):1628-1636.

4. Cohen J, Wells D, Munné S. Removal of 2 cells from cleavage stage embryos is likely to reduce the efficacy of chromosomal tests that are used to enhance implantation rates. Fertil Steril 2007 Mar;87(3):496-503.

5. de Boer KA, Catt JW, Jansen RPS, Leigh D, McArthur S. Moving to blastocyst biopsy for preimplantation genetic diagnosis and single embryo transfer at Sydney IVF. Fertil Steril 2004 Aug;82(2):295-298.

6. Kokkali G, Vrettou C, Traeger-Synodinos J, Jones GM, Cram DS, Stavrou D, Trounson AO, Kanavakis E, Pantos K. Birth of a healthy infant following trophectoderm biopsy from blastocysts for PGD of $\beta$-thalassaemia major: Case report. Hum Reprod 2005 Jul;20(7):1855-1859.

7. Capalbo A, Rienzi L, Cimadomo D, Maggiulli R, Elliott T, Wright G, Nagy ZP, Ubaldi FM. Correlation between standard blastocyst morphology, euploidy and implantation: an observational study in two centers involving 956 screened blastocysts. Hum Reprod 2014 Jun;29(6):1173-1181.

8. Hardarson T, Landuyt LV, Jones G. The blastocyst. Hum Reprod 2012;27(S1):i72-i91.

9. Taylor T, Patrick J, Gitlin S, Wilson J, Crian J, Griffin D. Outcomes of blastocysts biopsied and vitrified once versus those cryopreserved twice for euploid blastocyst transfer. Reprod Biomed Online 2014 Jul;29(1):59-64.

10. Wininger JD, Taylor TH, Orris JJ, Glassner M, Anderson SH. Pregnancy after rebiopsy and vitrification of blastocysts following allele dropout after day 3 biopsy. Fertil Steril 2011 Mar;95(3):1122.e1-1122.e2.

11. Greco E, Biricik A, Cotarelo R, Lammarone E, Rubion P, Tesarik J, Fiorentino F, Minasi M. Successful implantation and live birth of a healthy boy after triple biopsy and double vitrification of oocyte-embryo-blastocyst. SpringerPlus 2015 Jan;4:22.

12. Goossens V, De Rycke M, De Vos A, Staessen C, Michiels A, Verpoest W, Van Steirteghem A, Bertrand C, Liebaers I, Devroey P, et al. Diagnostic efficiency, embryonic development 
and clinical outcome after the biopsy of one or two blastomeres for preimplantation genetic diagnosis. Hum Reprod 2008 Mar;23(3):481-492.

13. Gleicher N, Kushnir VA, Barad DH. Preimplantation genetic screening (PGS) still in search of a clinical application: a systematic review. Reprod Biol Endocrinol 2014;12:22.
14. Gleicher N, Orvieto R. Is the hypothesis of preimplantation genetic screening (PGS) still supportable? A review. J Ovarian Res 2017;10:21.

15. Capalbo A, Ubaldi F, Rienzi L, Scott R, Treff N. Detecting mosaicism in trophectoderm biopsies: current challenges and future possibilities. Hum Reprod 2017 Mar;32(3):492-498. 